\title{
BMJ Open Acupuncture for patients with Alzheimer's disease: a systematic review protocol
}

\author{
Jing Zhou, ${ }^{1,2}$ Weina Peng, ${ }^{1}$ Wang Li, ${ }^{1,2}$ Zhishun Liu ${ }^{1}$
}

To cite: Zhou J, Peng W, Li W, et al. Acupuncture for patients with Alzheimer's disease: a systematic review protocol. BMJ Open 2014:4:e005896.

doi:10.1136/bmjopen-2014005896

- Prepublication history for this paper is available online. To view these files please visit the journal online (http://dx.doi.org/10.1136/ bmjopen-2014-005896).

Received 11 June 2014 Revised 18 July 2014 Accepted 1 August 2014
CrossMark

\footnotetext{
${ }^{1}$ Department of Acupuncture, Guang'anmen Hospital, China Academy of Chinese Medical Sciences, Beijing, China ${ }^{2}$ Beijing University of Chinese Medicine, Beijing, China
}

Correspondence to Professor Zhishun Liu; liuzhishun@aliyun.com

\section{ABSTRACT \\ Introduction: The aim of this protocol is to provide the methods used to assess the effectiveness and safety of acupuncture for the treatment of patients with Alzheimer's disease.}

Methods and analysis: We will search the following electronic databases: The Cochrane Library, PubMed, Medline, Embase, PsycINFO, Chinese Biomedical Literature Database, Chinese Medical Current Contents and China National Knowledge Infrastructure without restriction of language and publication status. Other sources such as Chinese acupuncture journals and the reference list of selected studies will also be searched. After screening the studies, a meta-analysis of randomised controlled trials will be conducted, if possible. Results expressed as risk ratios for dichotomous data and standardised or weighted mean differences for continuous data, will be used for data synthesis.

Dissemination: The protocol of this systematic review will be disseminated in a peer-reviewed journal and presented at a relevant conference.

Trial registration number PROSPERO CRD42014009619

\section{INTRODUCTION}

Alzheimer's disease (AD) is a progressive brain disorder characterised by neuropathological and neurochemical features. ${ }^{1}{ }^{2}$ The typical pathological characteristics of $\mathrm{AD}$ are degeneration of specific nerve cells, presence of neuritic plaques and neurofibrillary tangles. ${ }^{1}$ People with $\mathrm{AD}$ experience memory loss, cognitive impairment, difficulty communicating and mood changes, all of which will worsen over time. ${ }^{3}$ Disability and dependence because of $\mathrm{AD}$ place a financial burden on caregivers. ${ }^{4}$

$\mathrm{AD}$ is slightly more common in women than in men, ${ }^{5}$ and its prevalence doubles every 5 years, with higher frequencies among those aged $>85$ years. ${ }^{6}$ Experts estimated there were about 24.3 million cases of dementia in 2001 among the 14 WHO regions. The number of cases was predicted

\section{Strengths and limitations of this study}

- Our systematic review will provide a comprehensive, objective and systematic assessment of the use of acupuncture for the treatment of Alzheimer's disease.

- The results of this systematic review will help clinicians make decisions about treatment, and also provide clues for researchers in this subject area.

- The results of our review may help to expand the treatment options for patients with Alzheimer's disease.

- Because of the heterogeneity arising from various evaluations and reports of adverse reaction outcomes in studies, we will only describe the adverse reactions occurring during the treatment. The small trial sample sizes will also affect the comprehensive estimation of the risk of harm.

to reach 81.1 million by 2040 , with about 4.6 million new cases appearing every year. ${ }^{7}$ The worldwide societal costs of dementia disorders were estimated at US\$604 billion in 2010, which comprises about $1 \%$ of the aggregated global gross domestic product and has an enormous socioeconomic impact. $^{8}$

There are 10 common diagnosis criteria for AD. The criteria of the Diagnostic and Statistical Manual of Mental Disorders 4th edition (DSM-IV), the National Institute of Neurological and Communicative Disorders and Stroke and the $\mathrm{AD}$ and Related Disorders Association (NINCDS-ADRDA) criteria are probably the most widely used. ${ }^{9}$ The NINCDS-ADRDA-RC ${ }^{10}$ includes distinctive and reliable biomarkers for MRI and positron emission tomography for the diagnosis of AD. The National Institute on Aging and the Alzheimer's Association (NIA-AA) developed research criteria to capture the earliest stages, before full-blown dementia, as well as the full spectrum of the illness. ${ }^{11}$ Moreover, there is a Chinese version of 
clinical criteria for $\mathrm{AD}$, the operational criteria for the diagnosis of $\mathrm{AD}$ (OCDAD). ${ }^{12}$ Because of cultural and regional differences, the understanding and diagnosis of $\mathrm{AD}$ becomes more challenging, which can affect the outcomes of clinical studies.

Medical treatment of $\mathrm{AD}$ can improve symptoms, but it cannot halt or reverse its progression. Cholinesterase inhibitors are commonly used. Donepezil, rivastigmine and galantamine are approved by the Food and Drug Administration to treat the symptoms of mild to moderate $\mathrm{AD}$. However, raised costs and increased risk of serious adverse events associated with cholinesterase inhibitor treatment have been reported. ${ }^{13}$ Further, the effectiveness of memantine was demonstrated for the treatment of moderate to severe $\mathrm{AD}$ and its use in combination with donepezil ${ }^{14}$ resulted in a significantly slower progression of $\mathrm{AD}$ symptoms after long-term treatment. ${ }^{15}$ Additionally, nicergoline is used in AD because it increases cerebral blood flow and, consequently, reduces vascular resistance. ${ }^{16}$ Nevertheless, no nonpharmacological or pharmacological treatments prevent or cure this disease and the pharmacological interventions have been associated with many adverse events. ${ }^{17}$

Acupuncture originated thousands of years ago and it plays a vital part in traditional Chinese medicine. In recent years, acupuncture has been widely used by researchers in clinical practice for the treatment of $\mathrm{AD}$, with fewer associated adverse events occurring. ${ }^{18}$ Some studies in patients with $\mathrm{AD}$ who underwent scalp, Xiu Sanzhen manipulation and body acupuncture showed improved Mini Mental State Examination (MMSE) scores, activities of daily living and the hierarchic dementia scale. ${ }^{19}{ }^{20}$ According to our pre-search, nearly 20 randomised control trials have been published within the past 5 years and we consider it is necessary to reassess the efficacy and safety of acupuncture for the treatment of $\mathrm{AD}$.

\section{METHODS AND ANALYSIS}

\section{Inclusion criteria for study selection}

Types of studies

Randomised controlled trials without any restrictions of language and publication type are eligible for inclusion.

\section{Types of participants}

Studies evaluating people diagnosed with $\mathrm{AD}$ will be included regardless of their age, sex, ethnicity, education or economic status and whether or not they were living in their own homes or in a residential care facility or hospital, according to following criteria:
1. International Classification of Diseases version- $10^{2}$
2. $\mathrm{DSM}-\mathrm{IV}^{5}$
3. NINCDS-ADRDA criteria $^{1}$
4. NINCDS-ADRDA-RG ${ }^{10}$
5. NIA-AA workgroup criteria $^{11}$
6. OCDAD. ${ }^{12}$

If studies also include participants with other forms of dementia, then they will only be included if the patients with $\mathrm{AD}$ are reported separately. If the diagnostic criteria are not been clearly stated, trial authors will be contacted for clarifications. We will also include those studies that have applied different versions of the diagnostic criteria listed above because different organisations have often issued revised or updated versions.

\section{Types of interventions}

Studies reporting any type of acupuncture treatment will be included (body acupuncture, scalp acupuncture, auricular acupuncture, electroacupuncture, fire needling, elongated needle, intradermal needling). Point injection, laser acupuncture, tap-pricking will be excluded. We will exclude trials mainly comparing different acupuncture points of acupuncture or comparing different types of acupuncture.

Comparison interventions, including placebo control, sham acupuncture, no treatment, waiting list control, Western medicine, usual care and other conventional treatments, will also be included. Additionally, studies evaluating acupuncture combined with another treatment compared with that other treatment alone will also be included.

\section{Types of outcome assessments}

1. Cognitive function

2. Changes in global disease severity

3. Activities of daily living

4. Clinical global impression

5. Behaviour

6. Death

7. Mood

8. Safety as measured by incidence and severity of adverse effects

9. Dependency (such as institutionalisation)

10. Acceptability of treatment as measured by withdrawal from trials

11. Quality of life

12. Costs.

As for the outcome assessments, studies which applied scales such as MMSE, CDR (clinical dementia rating), CIBIC-plus (clinicians' interview-based impression of change-plus), NPI (neuropsychiatric inventory), etc, related to the types of outcome assessment domains listed above will be included.

\section{Search methods for identification of studies \\ Electronic searches}

We will search the following electronic databases without restriction of language and publication status from inception to 2014: Cochrane Central Register of Controlled Trials (The Cochrane Library), PubMed, Medline, Embase (Excerpta Medica Database), PsycINFO, Chinese Biomedical Literature Database, Chinese Medical Current Contents and China National Knowledge Infrastructure. 
We will use the following search terms: $\mathrm{AD}$, Alzheimer, dementia, acupuncture, body acupuncture, scalp acupuncture, auricular acupuncture, electroacupuncture, fire needling, elongated needle and intradermal needling. A Chinese translation of the same search terms will be used to search in the Chinese databases. The search strategy for PubMed is shown in table 1.

\section{Other sources}

The following journals published in Chinese will be searched: Chinese Acupuncture and Moxibustion (19812014), Journal of Clinical Acupuncture and Moxibustion (1985-2014), Journal of Traditional Chinese Medicine (1960-2014), New Journal of Traditional Chinese Medicine (1969-2014), Shanghai Journal of Acupuncture and Moxibustion (1982-2014) and Research of Acupuncture and Moxibustion (1976-2014). Relevant references cited in the studies selected will also be searched as supplementary sources. Conference proceedings and unpublished literature will also be included.

\section{Data collection and analysis}

Selection of studies

Two authors (JZ and WL) will review and screen the titles and abstracts to identify eligible trials according to

\begin{tabular}{|c|c|}
\hline No & Search items \\
\hline 1 & Randomised controlled trial \\
\hline 2 & Controlled clinical trial \\
\hline 3 & Randomised \\
\hline 4 & Randomly \\
\hline 5 & Placebo \\
\hline 6 & Trial \\
\hline 7 & 1 or $2-6$ \\
\hline 8 & Dementia \\
\hline 9 & Alzheimer's disease \\
\hline 10 & Cognitive disorders \\
\hline 11 & Cognitive impairment \\
\hline 12 & dement $^{\star}$ \\
\hline 13 & Alzheimer* \\
\hline 14 & 8 or $9-13$ \\
\hline 15 & Acupuncture therapy \\
\hline 16 & Acupuncture \\
\hline 17 & Acupoints \\
\hline 18 & Acupuncture* \\
\hline 19 & Body acupuncture \\
\hline 20 & Scalp acupuncture \\
\hline 21 & Auricular acupuncture \\
\hline 22 & Electroacupuncture \\
\hline 23 & Fire needling \\
\hline 24 & Elongated needle \\
\hline 25 & Intradermal needling \\
\hline 26 & 15 or $16-25$ \\
\hline 27 & 7 and 14 and 26 \\
\hline
\end{tabular}

the inclusion criteria; the full text will be read if necessary. Excluded studies will be listed in a table with the reasons for their exclusion. Any discrepancies will be resolved by discussion with ZL.

\section{Data extraction and management}

Two authors (JZ and WL) will extract data on participants, randomisation, interventions, outcomes, duration, follow-up and reasons for discontinuations, number of treatment-related adverse events, author information and interesting conflicts, independently using a data extraction form and then we will enter the data into Review Manager software (RevMan V.5.2.1). Different opinions will be discussed and missing data will be obtained by contacting trial authors for more information.

According to the different tolerance of participants and techniques of the acupuncturists, heterogeneity may exist in performing a meta-analysis of adverse reaction data. Adverse reactions will be summarised qualitatively according to the following three aspects: (1) number of fainting or other symptoms during acupuncture; (2) number of haematomas during treatment and (3) number of local infections.

\section{Assessment of risk of bias in included studies}

The Cochrane Collaboration Risk of Bias Tool $^{21}$ will be used to assess the methodological quality by the two authors (WL and JZ). The risk of bias in included studies will be evaluated according to the six domains: sequence generation, allocation concealment, blinding, incomplete data assessment, selective outcome reporting, other sources of bias. Other sources of bias may be caused by the different characteristics and representativeness of participants and by selective outcome reports due to conflict of interest. Additionally, based on these domains, the included studies will be classified into three categories: low risk, high risk and unclear. If there are insufficient or unclear items affecting the judgement of risk of bias, the study authors will be contacted for more details. Any disagreement will be resolved by discussion with ZL.

In acupuncture, blinding of acupuncturists is impossible, but for the studies using sham or placebo acupuncture as a control treatment, the assessment of blinding in both participants and outcome assessors can be conducted. For those studies in which blinding of participants is difficult-for example, those comparing acupuncture with another type of treatment, we will only assess blinding of the outcome assessors.

\section{Measures of treatment effect}

For dichotomous data, risk ratio with corresponding 95\% CIs will be used while continuous data will be expressed as mean differences with $95 \%$ CIs. Weighted mean differences will be used for data measured on the same scales and for which the same units are used; otherwise, standardised mean differences will be used. 
Dealing with missing data

We will attempt to collect additional information by contacting authors of included studies with missing data. If we fail to obtained sufficient data, the trials with missing data will be omitted from the data synthesis. An intention-to-treat analysis will be performed, if possible (the analysis should include the data of all the participants in the groups to which they were originally randomly assigned), and a sensitivity analysis will be used to determine whether the results are inconsistent.

\section{Assessment of heterogeneity}

We will search for overlapping CIs in forest plots and use a $\chi^{2}$ test for statistical heterogeneity and the $\mathrm{I}^{2}$ statistic $\left(\mathrm{I}^{2}>50 \%\right.$ shows the existence of heterogeneity) to estimate the level of heterogeneity across the studies.

\section{Assessment of reporting biases}

We will use funnel plots to detect potential reporting biases and small-study effects. The Egger method ${ }^{22}$ will be used to explain the asymmetry if more than 10 studies are included in the meta-analysis.

\section{Data synthesis}

If it is possible to carry out a meta-analysis, RevMan V.5.2.1 software will be used to combine the relative risks for dichotomous outcomes and mean differences for continuous outcomes both with $95 \%$ CIs. We will use the fixed-effect model if there is no evidence of heterogeneity; otherwise, we will apply a random-effects model and reach a conclusion more cautiously. If significant heterogeneity between studies is found, we will search for the possible causes from both clinical and methodological perspectives and provide an explanation or conduct subgroup analysis. Finally, if there is substantial heterogeneity between studies, we will conduct a descriptive analysis.

\section{Subgroup analysis}

A subgroup analysis will be performed based on the type of acupuncture intervention (body acupuncture, scalp acupuncture, auricular acupuncture, electroacupuncture, fire needling, elongated needle, intradermal needling,) because this is the main factor causing heterogeneity. Additionally, the duration of treatment and combination of treatment (acupuncture alone or acupuncture with another treatment) will also be considered. Furthermore, sample sites will be classified based on the place of participants' residence (such as own homes, a residential care facility or hospitals), and the different progression of $\mathrm{AD}$ will also be considered and classified into three categories: mild, moderate and severe.

\section{Sensitivity analysis}

We will conduct a sensitivity analysis to verify the robustness of the study conclusions, assessing the impact of methodological quality, study design, sample size and the effect of missing data as well as the analysis methods on the result of this review. ${ }^{23}$ We will also use sensitivity analyses to investigate suspected funnel plot asymmetry.

\section{Ethics and dissemination}

Ethical approval will not be needed because the data used in this systematic review will not be individual patient data and there will be no concerns about privacy. The results will be disseminated by its publication of the manuscript in a peer-reviewed journal or presented at a relevant conference.

\section{DISCUSSION}

A systematic review of acupuncture for $\mathrm{AD}$ published in 2009 analysed three randomised control trials. In the previous review, type of participants, outcome assessment and other details of the methodology were not clearly and specifically described. The serious methodological flaws did not allow the authors to draw convincing conclusions. Furthermore, the researchers did not calculate the proportion of treatment-related adverse events. ${ }^{24}$ Thus, a comprehensive and objective systematic

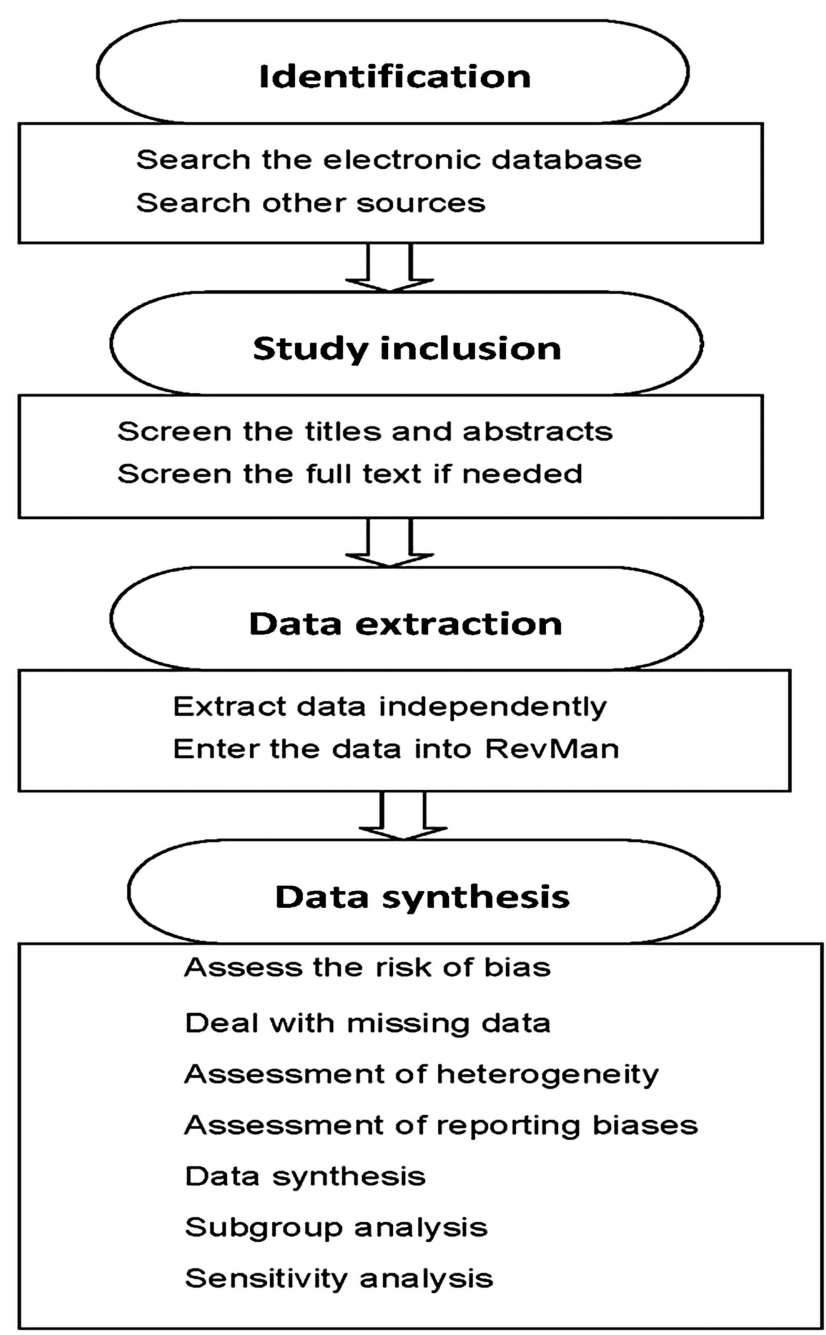

Figure 1 Process of the systematic review. 
review is needed. The process of performing this systematic review, shown in figure 1, will be separated into four parts: identification, study inclusion, data extraction and data synthesis. Collection of data is continuing for our study. We consider that this review will assist clinicians during the decision-making process when treating patients with $\mathrm{AD}$ and will also provide clues for researchers in this subject area. At the same time, patients with $\mathrm{AD}$ may benefit from additional treatment alternatives.

Contributors JZ and ZL contributed to the conception of the study. The manuscript protocol was drafted by JZ and was revised by WP. The search strategy was developed by all the authors and will be performed by $\mathrm{JZ}$ and WL, who will also independently screen the potential studies, extract data from the included studies, assess the risk of bias and complete the data synthesis. ZL will arbitrate in cases of disagreement and ensure the absence of errors. All authors approved the publication of the protocol.

\section{Competing interests None.}

Provenance and peer review Not commissioned; externally peer reviewed.

Open Access This is an Open Access article distributed in accordance with the Creative Commons Attribution Non Commercial (CC BY-NC 4.0) license, which permits others to distribute, remix, adapt, build upon this work noncommercially, and license their derivative works on different terms, provided the original work is properly cited and the use is non-commercial. See: http:// creativecommons.org/licenses/by-nc/4.0/

\section{REFERENCES}

1. McKhann G, Drachman D, Folstein M, et al. Clinical diagnosis of Alzheimer's disease: report of the NINCDS-ADRDA Work Group under the auspices of Department of Health and Human Services Task Force on Alzheimer's disease. Neurology 1984;4:939-44.

2. World Health Organization. The ICD-10 Classification of Metal and Behavioural Disorders: Clinical Descriptions and Diagnostic Guidelines. 1992.

3. Prince PM, Prina M, Guerchet DM. World Alzheimer Report: Journey of Caring: an analysis of long-term care for dementia 2013:1-88.

4. Ferri $\mathrm{CP}$, Prince $\mathrm{M}$, Brayne $\mathrm{C}$, et al. International global prevalence of dementia: a Delphi consensus study. Lancet 2005;366:2112-17.

5. American Psychiatric Association. Diagnostic and Statistical Manual of Mental Disorders, 4th text version (DSM-IV). Washington, DC: APA, 2000.

6. Galton CJ, Hodeges JR. The spectrum of dementia and its treatment. J R Coll Physicians Lond 1999;33:234-9.

7. Prince M, Brycea R, Albanesea E, et al. The global prevalence of dementia: a systematic review and metaanalysis. Alzheimers Dement 2013;9:63-75.
8. Wimo A, Ballard C, Brayne C, et al. Health economic evaluation of treatments for Alzheimer's disease: impact of new diagnostic criteria. J Intern Med 2014;275:304-16.

9. Hogervorst E, Bandelow $\mathrm{S}$, Combrinck $\mathrm{M}$, et al. The validity and reliability of 6 sets of clinical criteria to classify Alzheimer's disease and vascular dementia in cases confirmed post-mortem: added value of a decision tree approach. Dement Geriatr Cogn Disord 2003;16:170-80.

10. Dubois $\mathrm{B}$, Feldman $\mathrm{HH}$, Jacova $\mathrm{C}$, et al. Research criteria for the diagnosis of Alzheimer's disease: revising the NINCDS-ADRDA criteria. Lancet Neurol 2007;6:734-46.

11. McKhann GM, Knopman DS, Chertkow $\mathrm{H}$, et al. The diagnosis of dementia due to Alzheimer's disease: recommendations from the National Institute on Aging and the Alzheimer's Association workgroups on diagnostic guidelines for Alzheimer's disease. Alzheimers Dement 2011;7:263-9.

12. Tian JZ, Shi J, Wei MQ, et al. A Chinese version of clinical criteria for the diagnosis of Alzheimer's disease. Chin J Front Med Sci 2012;4:1-6.

13. Zec RF, Burkett NR. Non-pharmacological and pharmacological treatment of the cognitive and behavioral symptoms of Alzheimer's disease. NeuroRehabilitation 2008;23:425-83.

14. Tampi RR, van Dyck $\mathrm{CH}$. Memantine: efficacy and safety in mild-to-severe Alzheimer's disease. Neuropsychiatr Dis Treat 2007;3:245-58.

15. Atri A, Shaugnessy LW, Locascio JJ, et al. Long-term course and effectiveness of combination therapy in Alzheimer's disease. Alzheimer Dis Assoc Disord 2008;22:209-1.

16. Winblad B, Fioravanti M, Dolezale T, et al. Therapeutic use of nicergoline. Clin Drug Investig 2008;28:533-52.

17. Hansen R, Gartlehner G, Webb PA. Efficacy and safety of donepezil, galantamine and rivastigmine for the treatment of Alzheimer's disease: a systematic review and meta-analysis. Clin Interv Aging 2008;3:211-55.

18. Luo $Q, D u Y$. Clinical research progress of acupuncture to treat Alzheimer's disease. Liaoning J Tradit Chin Med 2014;41:594-6.

19. Cheng $\mathrm{H}, \mathrm{Yu} \mathrm{J}$, Peng $\mathrm{Y}$, et al. Clinical research progress of acupuncture to treat Alzheimer's disease. Liaoning $J$ Tradit Chin Med 2008;35:630-3.

20. Liu Z, Niu W, Yang X, et al. The clinical research of impact on cognitive function of patients with Alzheimer's disease by using Xiu Sanzhen. Shan Xi J Tradit Chin Med 2008;29:11-12.

21. Higgins JPT, Green S. Cochrane handbook for systematic review of intervention version 5.1.0 [updated March 2011]. The Cochrane Collaboration 2011. http://www.cochrane-handbook.org (accessed Jun 2014).

22. Deeks JJ, Higgins JPT, Altman DG. Analysing data and undertaking meta-analysis. In: Higgins JPT, Green S, eds. Cochrane Handbook for Systematic Reviews of Interventions Version 5.1.0 [updated March 2011]. The Cochrane Collaboration, 2011. http://www. cochrane-handbook.org (accessed Jun 2014).

23. Egger M, Davey Smith G, Schneider M, et al. Bias in meta-analysis detected by a simple, graphical test. BMJ 1997;315:629-4.

24. Lee MS, Shin BC, Ernst E. Acupuncture for Alzheimer's disease: a systematic review. Int J Clin Pract 2009;63:874-9. 